\title{
A split body trial comparing dilute bleach vs. dilute apple cider vinegar compresses for atopic dermatitis in Chicago: a pilot study
}

\begin{abstract}
Topical therapies such as dilute bleach and acetic acid are widely used to treat atopic dermatitis, though research regarding their efficacy and mechanism of action is lacking. The objective of this single-blinded, pilot study of eleven patients was to compare clinical and antibacterial benefits of bleach and apple cider vinegar compresses when locally applied to eczematous skin. While both solutions demonstrated comparable clinical benefit, as measured by improvement in local Eczema Area and Severity Index (EASI) scores, both similarly lacked efficacy with respect to decreasing the burden of Staphylococcus aureus on eczematous lesions. These results suggest that while bleach or ACV may be effective treatments for $\mathrm{AD}$, the mechanism of either solution is likely not due to antibacterial properties.
\end{abstract}

Keywords: atopic dermatitis, eczema, bleach bath, apple cider vinegar, staphylococcus aureus
Volume 3 Issue I - 2019

\author{
Neil R Lim,' Alison D Treister,' Vera Tesic, ${ }^{2}$ \\ Kachiu C Lee, ${ }^{3}$ Peter A Lio ${ }^{1,4}$ \\ 'Department of Dermatology, Northwestern University \\ Feinberg School of Medicine, USA \\ ${ }^{2}$ Department of Pathology, University of Chicago, USA \\ ${ }^{3}$ Department of Dermatology,Warren Brown Alpert Medical \\ School of Brown University, USA \\ ${ }^{4}$ Medical Dermatology Associates of Chicago, USA
}

Correspondence: Peter A Lio MD, Department of Dermatology, Feinberg School of Medicine, Northwestern University, 363 W Erie Street, Suite 350, Chicago, IL 60654, USA, Tel +I (3I2) 995 1955, Email peterlio@gmail.com

Received: January 24, 2018 | Published: February 04, 2019

Abbreviations: EASI, eczema area and severity index; $\mathrm{AD}$, atopic dermatitis; ACV, apple cider vinegar; TEWL, transepidermal water loss; RODAC, replicate organism detection and counting

\section{Introduction}

Atopic dermatitis (AD) is a common skin disorder that is frequently complicated by bacterial colonization and secondary infection with Staphylococcus aureus. Evidence suggests that the density of $S$. aureus colonization correlates with disease severity, and is increased during flares. ${ }^{1}$ Dilute bleach baths are often employed as adjunctive therapy for $\mathrm{AD}$, as these baths are thought to potentially improve disease severity via the antimicrobial properties of sodium hypochlorite, bleach's active ingredient. The efficacy of bleach baths have been recently been called into question, ${ }^{2}$ their use remains controversial and some even attribute their clinical benefit to anti-inflammatory or other effects. Interestingly, the efficacy of bleach may be due to hypochlorous acid, which forms when sodium hypochlorite is added to water. This acid when applied topically has been shown to decrease pro-inflammatory cytokines comparable to tofacitnib, a Janus kinase inhibitor. ${ }^{3}$ Given that some patients are averse to bleach, disinfectants such as acetic acid have been suggested as an alternative strategy, however, evidence for their use is currently lacking. We sought to compare the effectiveness of dilute bleach to dilute apple cider vinegar (ACV), hypothesizing that ACV would exhibit comparable reduction of $S$. aureus skin burden and clinical improvement compared to bleach.

\section{Methods}

Following approval by the New England Institutional Review Board, patients with moderate-severe $\mathrm{AD}$ and clinical signs of $\mathrm{AD}$ of varying severity in the bilateral antecubital fossae were recruited from the Medical Dermatology Associates of Chicago outpatient clinic from February to July 2017. Eleven subjects were screened and enrolled with a median age of 33 (range 21-49); seven were female. Eight subjects were Caucasian, and three were Asian. Each subject was randomized to receive bleach on the antecubital fossa of one arm, and
ACV on the other arm. Subjects were instructed to apply a compress saturated with a pre-prepared dilute bleach solution $(0.005 \%$ sodium hypochlorite) to the antecubital fossa of one arm for ten minutes, then to apply a cloth saturated with dilute ACV ( $0.5 \%$ acetic acid) to the opposite arm for another ten minutes. This regimen was repeated once daily for 14 days. Patients were permitted to continue using emollients and previously prescribed $\mathrm{AD}$ therapies, but were instructed not to initiate any new treatments during the study period.

Subjects' bilateral antecubital fossae were cultured for $S$. aureus via the use of Replicate Organism Detection and Counting (RODAC TSA II) plates containing trypticase soy agar with $5 \%$ sheep blood at baseline and at follow-up after 14 days. Subjects' antecubital fossae were also assessed clinically via the local Eczema Area and Severity Index (EASI) score at both time points. A single dermatologist (PAL) performed all clinical evaluations at baseline and at 14 days while blinded to which arm had received which treatment group. The local EASI score was calculated based on four signs (erythema, edema/ papulation, excoriation, and lichenification) assessed at the target area; the severity of each sign was graded from 0-3 (most severe) and added for a total score of 0-12. A Wilcoxon rank sum test was used to compare EASI scores between fellow arms (Stata version 13; College Station, TX). A p-value less than 0.05 was considered statistically significant.

\section{Results}

There were no significant differences in outcomes between arms treated with bleach and ACV. Eight out of the 11 subjects (73\%) improved by at least 1 point by local EASI score on their bleach arms, and 9 out of 11 subjects $(82 \%)$ improved by at least one point on their ACV arms (Table 1). Only one subject worsened after treatment, and deterioration was noted at both antecubital fossae in spite of either regimen. There was no statistical significance for mean improvement in clinical severity when comparing the two interventions: bleach improved EASI scores by an average of 2.1 points, while ACV improved EASI scores by an average of 1.7 points $(\mathrm{p}=0.773)$. 
Table I Local EASI Scores at Baseline and Follow-Up

\begin{tabular}{lllll}
\hline & \multicolumn{2}{l}{ Dilute Bleach Arm } & \multicolumn{2}{l}{ Dilute ACV Arm } \\
\cline { 2 - 5 } & Baseline & Follow-Up & Baseline & Follow-Up \\
\hline Subject I & 2 & I & 2 & I \\
Subject 2 & I & 0 & 2 & I \\
Subject 3 & I & 0 & I & 0 \\
Subject 4 & 4 & 6 & 3 & 6 \\
Subject 5 & 4 & 1 & 2 & 1 \\
Subject 6 & 6 & 5 & 6 & 5 \\
Subject 7 & 3 & 3 & 3 & 3 \\
Subject 8 & 10 & 4 & 10 & 4 \\
Subject 9 & 4 & 0 & 4 & 2 \\
Subject 10 & I & 1 & 1 & 0 \\
Subject I 1 & 10 & 2 & 10 & 2 \\
\hline
\end{tabular}

Only one subject (Subject 11) had clearance of S. aureus, which occurred in both antecubital fossae after 14 days, while two other subjects (Subjects 4 and 7) actually developed $S$. aureus colonization bilaterally while on the treatment regimens. Five subjects were not colonized at baseline and remained clear at 14 days, while three subjects were colonized at baseline and remained colonized despite the interventions (Table 2).

Table 2 Staphylococcus aureus Colonization Status at Baseline and Follow-Up

\begin{tabular}{cllll} 
& Baseline & \multicolumn{3}{c}{ Follow-Up } \\
\cline { 2 - 5 } & R Arm & LArm & R Arm & L Arm \\
\hline Subject I & None & None & None & None \\
Subject 2 & MRSA & MRSA & MRSA & MRSA \\
Subject 3 & None & None & None & None \\
Subject 4 & None & None & MSSA & MSSA \\
Subject 5 & None & None & None & None \\
Subject 6 & MRSA & MRSA & MRSA & MRSA \\
Subject 7 & None & None & MSSA & MSSA \\
Subject 8 & MSSA & MSSA & MSSA & MSSA \\
Subject 9 & None & None & None & None \\
Subject 10 & None & None & None & None \\
Subject I & MRSA & MRSA & None & None \\
& & &
\end{tabular}

MSSA, methicillin-sensitive Staphylococcus aureus; MRSA, methicillin-resistant Staphylococcus aureus

There were no significant adverse events; three subjects noted mild itching, stinging, and dryness with $\mathrm{ACV}$, while one subject noted mild irritation with bleach. Six patients reported preferring bleach to ACV after the study's conclusion, all of whom cited ACV's pungent smell as the deciding factor. Of note, five subjects failed to complete the full treatment regimen for at least one day out of fourteen, attributed in all cases to these subjects either simply forgetting to perform it, or traveling out of town.

\section{Discussion}

While clinicians have employed bleach baths as add-on therapy for $\mathrm{AD}$ for quite some time, the mechanism for improving disease severity remains unclear. Though tempting to attribute its benefits to its antimicrobial properties, recent evidence suggests that bleach is not effective as an antimicrobial agent at all-at least at the dilute concentrations typically used in bleach baths. A study by PerezNavario et al. noted that twelve weeks of bleach baths resulted in reduction of disease severity, improvement in itch, and improvement in transepidermal water loss (TEWL) measurements - but had no effect on $S$. aureus colonization or on skin dysbiosis. ${ }^{4}$ Another study by Gonzalez et al..$^{5}$ compared topical corticosteroids alone versus in conjunction with bleach baths and found that the skin microbiome was normalized after 4 weeks in subjects from both treatment groupssuggesting that bleach baths conferred no added benefit in restoring the cutaneous microbiome. ${ }^{5}$ Most intriguingly, a randomized, doubleblinded, cross-over trial of 40 children conducted by Hon et al. ${ }^{6}$ demonstrated no significant differences between bleach baths versus water baths in reducing $S$. aureus colonization - and even observed a greater reduction in SCORAD scores with water baths compared to bleach $(\mathrm{p}=0.03) .{ }^{6}$ Taken together, these results suggest that bleach baths improve disease severity not via antimicrobial action, but rather by some other mechanism - perhaps by improving the skin barrier to reduce transepidermal water loss, reducing itch, decreasing inflammation, ${ }^{7}$ or even simply through gentle hydration via consistent bathing combined with emollients.

$\mathrm{ACV}$ baths have yet to be investigated as thoroughly as bleach baths, but offer a similarly plausible mechanism for improving AD. Various studies have demonstrated the helpfulness of dressings saturated with $0.5-5 \%$ acetic acid (the active ingredient in ACV) in not only assisting with chronic and superficial wound healing, but also in eradicating bacterial colonization. ${ }^{8,9}$ Interestingly, a recent retrospective cohort study of pediatric $\mathrm{AD}$ patients found that neither dilute bleach nor acetic vinegar baths led to a decrease in subsequent use of systemic antibiotics over one year, suggesting that neither of these topical therapies exhibit anti-infective properties on eczematous skin. ${ }^{10}$ While ACV resulted in comparable improvement in local EASI scores compared to bleach in our cohort, its similarly unimpressive effect on $S$. aureus colonization suggests that any of its beneficial effects are likely not attributable to antimicrobial properties, either. While it has been hypothesized that ACV may work by altering the $\mathrm{pH}$ of atopic skin and restoring its barrier, ${ }^{11,12}$ further studies are needed to help clarify the mechanism underlying both ACV and bleach's action. From a clinical standpoint, it is encouraging to observe similar symptom relief with both ACV and bleach baths, but larger controlled trials are needed to rule out confounding contributions from the placebo effect and/or the therapeutic effect of bathing in general. The results of this pilot study suggest that

i. Topically-applied bleach and ACV exhibit similar efficacy in improving $\mathrm{AD}$, and that

ii. This benefit does not appear to be conferred by the theoretical antimicrobial properties of either agent.

Although these outcomes invite further inquiry into better understanding these interventions, it is encouraging to observe preliminarily that ACV offers comparable clinical benefits to bleach. A subset of patients cannot tolerate bleach, and ACV appears to be a similarly effective (though pungent) alternative that dermatologists may consider including in their toolkits for this group. 
It is important to note, however, that this study was limited by a small sample size, short treatment duration, the localized nature of the intervention compared to full submersion in a bath, and the absence of a placebo control. Further, patients were only of two ethnicities, recruited from a single center, and evaluated by a sole physician. Five patients failed to complete the full treatment regimen for at least one day out of fourteen. Seven out of 11 subjects were also not colonized with $S$. aureus even at baseline, resulting in a final pool of subjects that was insufficient to detect any meaningful effect of the interventions on $S$. aureus clearance, should one exist. Furthermore, treatment was limited to one concentration, leading to an inability to determine dosedependent effects. Lastly, given the relapsing-remitting course of AD, it is possible that the improvement in disease severity observed with both interventions could be attributed to the natural course of disease. Longer, multicenter, placebo-controlled trials with larger sample sizes are needed in order to better clarify the benefits of dilute ACV and bleach, as well as to elucidate the potential underlying mechanisms underlying them.

\section{Disclosures}

All authors have no conflicts of interest to declare.

\section{Funding}

This study was supported by a grant from La Foundation pour la Dermatite Atopique (Toulouse, France). The funder had no involvement in study design, data collection, data analysis, manuscript preparation, and/or publication decisions.

\section{Acknowledgments}

We would like to acknowledge Paula Mueller for her tireless work in managing the study logistics.

\section{Conflicts of interest}

Authors declare that there is no conflict of interest.

\section{References}

1. Bath-Hextall FJ, Birnie AJ, Ravenscroft JC, et al. Interventions to reduce Staphylococcus aureus in the management of atopic eczema: an updated Cochrane review. Br J Dermatol. 2010;163(1):12-26.
2. Chopra R, Vakharia PP, Sacotte R, et al. Efficacy of bleach baths in reducing severity of atopic dermatitis: a systematic review and metaanalysis. Ann Allergy Asthma Immunol. 2017;119(5):435-440.

3. Fukuyama T, Ehling S, Wilzopolski J, et al. Comparison of topical tofacitinib and $0.1 \%$ hypochlorous acid in a murine atopic dermatitis model. BMC Pharmacology and Toxicology. 2018;19:37.

4. Perez-Nazario N, Yoshida T, Fridy S, et al. Bleach baths significantly reduce itch and severity of atopic dermatitis with no significant change in S. aureus colonization and only modest effects on skin barrier function. Journal of Investigative Dermatology. 2015; Nature Publishing Group 75 Varick Street, $9^{\text {th }}$ FLR, New York, NY 10013-1917 USA. p. S37-S.

5. Gonzalez ME, Schaffer JV, Orlow SJ, et al. Cutaneous microbiome effects of fluticasone propionate cream and adjunctive bleach baths in childhood atopic dermatitis. J Am Acad Dermatol. 2016;75(3):481-493.

6. Hon Kl, Tsang YC, Lee VW, et al. Efficacy of sodium hypochlorite (bleach) baths to reduce Staphylococcus aureus colonization in childhood onset moderate-to-severe eczema: a randomized, placebocontrolled cross-over trial. J Dermatolog Treat. 2016;27(2):156-162.

7. Leung TH, Zhang LF, Wang J, et al. Topical hypochlorite ameliorates NF-kB-mediated skin diseases in mice. $J$ Clin Invest. 2013;123(12):5361-5370

8. Jeong HS, Lee BH, Lee HK, et ak. Negative pressure wound therapy of chronically infected wounds using $1 \%$ acetic Acid irrigation. Arch Plast Surg. 2015;42(1):59-67.

9. Ryssel H, Kloeters O, Germann G, et al. The antimicrobial effect of acetic acid-an alternative to common local antiseptics? Burns. 2009;35(5):695-700.

10. Asch S, Vork DL, Joseph J, et al. Comparison of bleach, acetic acid, and other topical anti-infective treatments in pediatric atopic dermatitis: A retrospective cohort study on antibiotic exposure. Pediatric dermatology. 2019;36(1):115-120.

11. Panther DJ, Jacob SE. The importance of acidification in atopic eczema: An underexplored avenue for treatment. J Clin Med. 2015;4(5):970-978.

12. Lee NR, Lee HJ, Yoon NY, et al. Acidic water bathing could be a safe and effective therapeutic modality for severe and refractory atopic dermatitis. Ann Dermatol. 2016;28(1):126-129. 